biochemically. The predicted dose of Pamidronate to achieve a normal alkaline phosphatase (ALP) and fastung urinary hydroxyproline/creatinine ratio (HYPRO) was calculated and administered as weekly intusions of enther 30 or $45 \mathrm{mg}$.

In all 10 patients in the previously untreated group. ALP fell into the upper normal range; there was a slight tendency for a greater than expected fall in ALP $(p<0.05)$. Onty $7 / 10$ patients achieved normal HYPRO values, but this tendency to underpredict the fall in HYPRO was not statistically signficam. For the 5 patients in the relapsing group, the actual reductions in ALP and HYPRO were much less than expected and none of these patients achieved biochemical remission.

In conclusion, the BPS model for predicting the dose of Pamidronate to achieve biochernical remission works well in prevously untreated patients. but not for patsents who are relapsing.

\section{BACTERLAL OVERGROWTH AND}

METHYLAMNE EXCRETION IN THE ELDERLY

M MAC MAHON, M HEALY*, RR O' MOORE*, CT KEANE ${ }^{\prime}$, JB WALSH and D COAKLEY

Mercer's Institute for Research on Ageing,

Depts. of Biochemistry* and Clinical Microbiology*,

St. James's Hospital, Dublin.

The bacterial by-products of choline metabolism are absorbed in the duodenum and excreted via the kidneys. It has been suggested that smail intestinal bacterial overgrowth (SIBO) may augment this process and produce toxic metabolites of choline; methylamines [Simenhoff et al. 1976, Lancet 2, 818-21]. Intestinal bacteria exposed to choline in vitro bave been shown to produce these metabolites [Johnson et al. 1977, Med. Lab. Sci. 34, 131-43].

As methylamines have the potential to form carcinogenic precursors. and to alter the bioavailibility of certain orally administered drugs, SIBO may therefore be implicated in these toxic processes.

Aims: (i) To determine if urinary dimethylamine (DMA), an endmetabolite of choline metabolism, is a reliable means of diagnosing SIBO, and (ii) to assess if the presence of certain small bowel organisms is associated with raised urinary DMA levels.

Methods: Urinary DMA ( $\mu \mathrm{mol} / \mathrm{l})$ was measured by gas liquid chromatography after choline ingestion, in a group of elderly patients: 18 culture proven SIBO, including 11 with strict anaerobes. and 7 culture negatives.

The presence of each of the bacterial species: Bacteroides, Clostridium, Enterobacteriaceae, Peptostreprococcus, Veillonell and Gram positive non-sporing rods was sndied in relation to DMA excretion.

Results; Urinary DMA; (i) did not differ between controls and the elderly patient group ( $P>0.05$ ), (ii) did not differ between the $S I B O$ and culture negative groups ( $P>0.05$ ), (iii) was not raised in the presence of small bowel anaerobes, and (iv) did not correlate significantly with total bacterial counts $(r=02)$, or, strict anaerobic counts $(r=0.1)$. The presence of Peprostreptococcus spp. was associated with the highest DMA production amongst the individual species $(\mathrm{P}<0.05)$.

Conclusion: Urinary DMA is not a reliable marker of SIBO and it is unlikety that methylamine production is increased as a result of SIBO in the elderly.
SCHEDULE for the EVALUATION of INDIVIDUAL QUALITY Of LIFE (SEQOL): ITS USE IN DEMENTIA

RF COEN*^, D OMAHONY^, CA O'BOYLE*, J BROWNE*, BA LAWLOR^, JB WALSH^. D COAKLEY^ \& CRB JOYCE+

Department of Psychology, Royal College of Surgeons in Ireland, Dublin 2*: Mercer's Institute for Research on Ageing, St. James' Hospital. Dublin 8^; and University Psychiatric Policlinic, Bern, Swizzerland ${ }^{+}$.

Many existing methods of measuring quality of life ( $Q \mathrm{OL})$ may be more accurately described as measures of health status or life satisfaction. Such measures generally impose external value systems on the individual. Judgement analysis (JA), denved from social judgement theory, permits individual judgements to be modelled mathematically. The Schedule for the Evaluation of Individual Quality of Life (SEIQLL) was developed by adapting $\mathrm{JA}$ to QoL measurement. allowing individuals to describe their QoL in terms of those factors which they consider important [O'Boyle er al. 1992, Lancet, 1088-1091]

Aims: The purpose of the present study was to evaluate the feasibility. validity and reliability of using the SEIQOL to measure the QoL of dementia patients.

Methods: The SEIQOL was administered to 20 patients meeting DSM-IIIR criteria for mild dementia (15 female, 5 male; mean age $=73$, range 67-81) Cognitive status was evaluated by CAMCOG from CAMDEX (mean=70.75, range 56-86)) and the Mini-Mental State Examination (mean $=22.75$, range 19-27).

Results: All patients were able to complete some elements of the SEIQOL but only 6 were willing or able to complete the full version. Mean CAMCOG scores distinguished this group of 6 patients from the remaining $14(t=3.5, p<0.01)$. Mean internal variance ( $\left.\mathbf{R}^{2}\right)$ provides one estimate of construct validity, and the internal reliability coefficient (r) provides a measure of judgement reliability. For the 6 patients who completed the SElQoL mean $R^{2}$ $=0.70(s d=0.11$, range $=0.57-0.83)$ and mean $r=0.74(s d=0.20$ range $=0.42-0.97$, both of which compare favourably with normative data

Conclusion: For dementia patients with mild cognitive impairment the SEIOOL can provide a feasible, valid and reliable measure of $\mathrm{O}$ LL. With increasing cognitive impairment patients are less likely to be able or willing to complete the SEIQOL.

ATRIAL FIBRILLATION IN THE ELDERLY: TO ANTICOAGULATE OR NOT?

D. KING, K. DAVIES, A. SLEE and J.H. SILAS

Clatterbridge Hospital. Wirral

The use of oral anticoagulants and aspirin for the primary prevention of stroke in elderly patients with atrial fibrillation. (AF), is controversial. To establish current practice a questionnaire was circulated to 300 geriatricians. (G) and 300 cardiologists (C). The response rate was $514 \mathrm{G}$ and $47 \% \mathrm{C}$. The table shows the of consultants who prescribe warfarin in conditions associated with AF in otherwise " $f$ it" elderly.

Diagnosis. (>70 years) $G(\xi) \quad C(\xi)$

Mixed Aortlc Valve

Disease (AVD)

Dilated Cardiompopa thy

(DCM)

37

$24 \mathrm{p}<0.05$

52

$86 \mathrm{p}<0.01$ 